Supporting Information for:

\title{
Effect of Polymer Topology and Residue Chirality on Biodegradability of Polypeptide Hydrogels
}

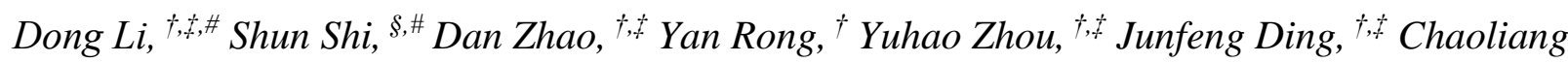
He, ${ }^{*}, \dot{t}, \dot{t}$ and Xuesi Chen ${ }^{*}, \dot{t}$

${ }^{\dagger}$ CAS Key Laboratory of Polymer Ecomaterials, Changchun Institute of Applied Chemistry, Chinese Academy of Sciences, Changchun, 130022, Jilin, P. R. China

¥University of Science and Technology of China, Hefei, 230026, Anhui, P. R. China

${ }^{\S}$ College of Polymer Science and Engineering, State Key Laboratory of Polymer Materials Engineering, Sichuan University, Chengdu, 610065, Sichuan, P. R. China

Corresponding Authors

*Email: clhe@ciac.ac.cn (C. He); xschen@ciac.ac.cn (X. Chen) 


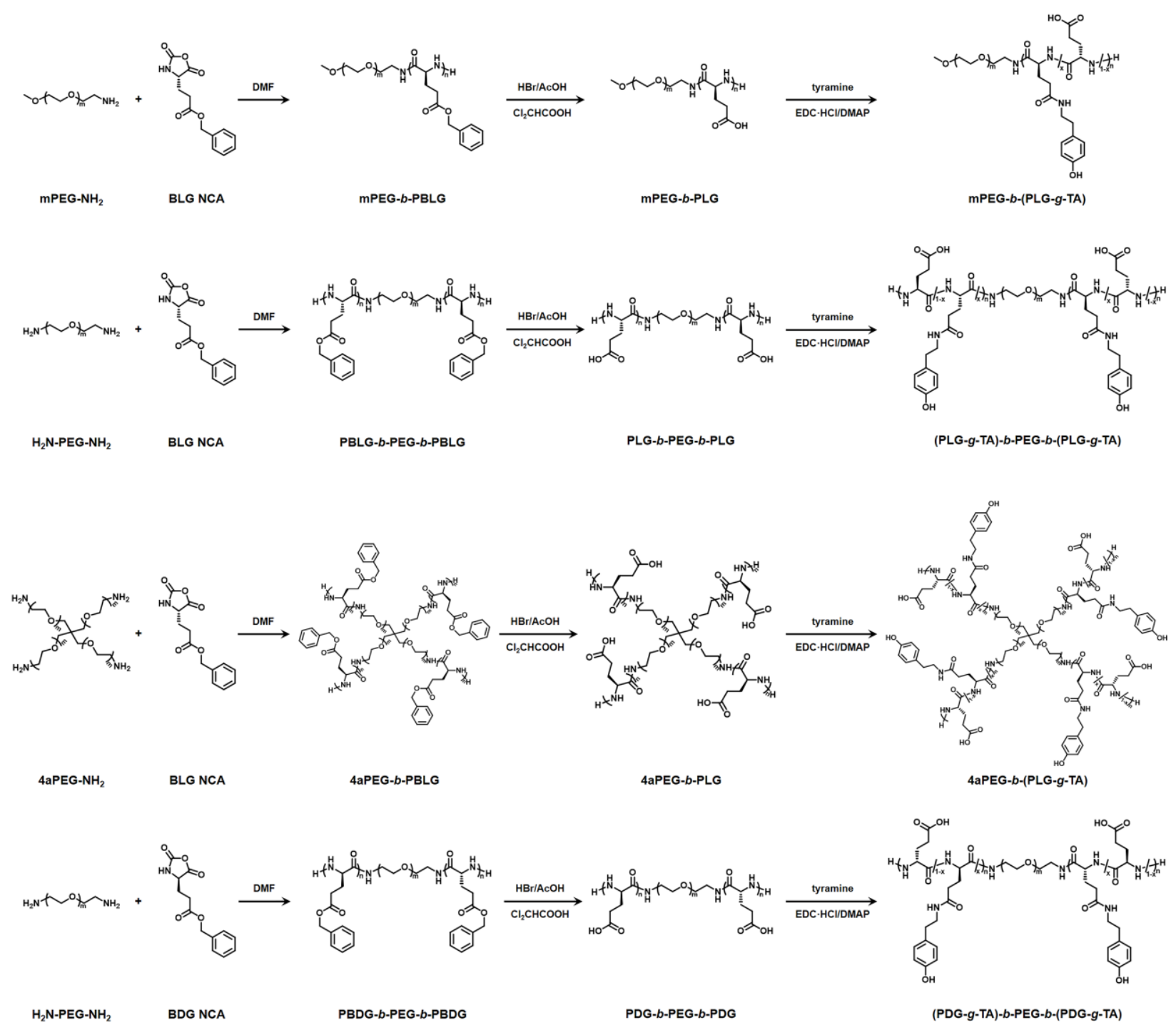

Scheme S1. Synthesis route of four types of PEG/(PGA- $g$-TA) copolymers with different polypeptide residue chirality and topologies. $m$ and $n$ indicate the amounts of repeating units within PEG and polypeptide segments, respectively, and $\mathrm{x}$ indicates the graft ratio of TA. 

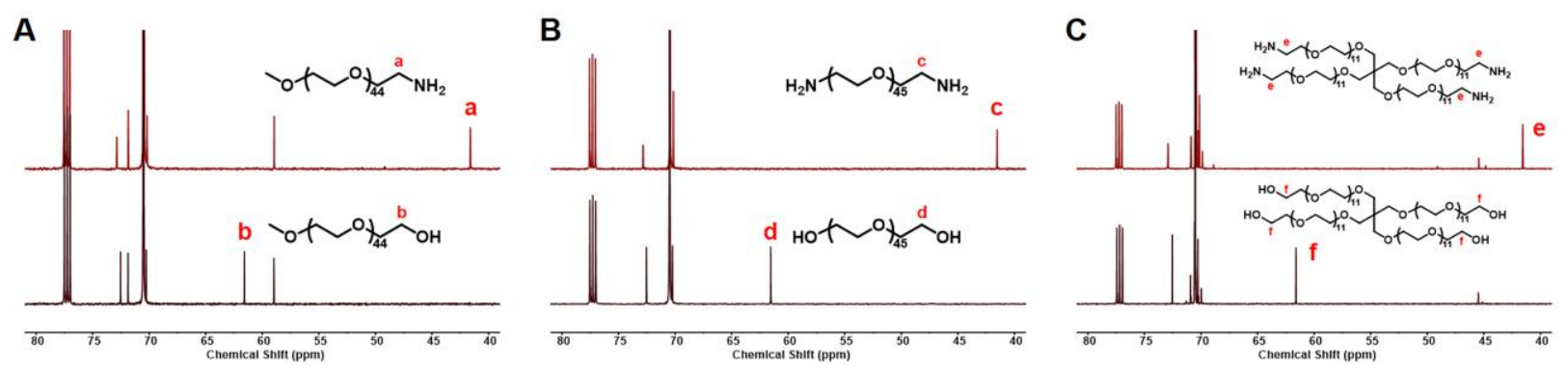

Figure S1. ${ }^{13} \mathrm{C}$ NMR spectra of hydroxyl- or amino-terminated PEGs $\left(500 \mathrm{MHz}, \mathrm{CDCl}_{3}\right)$. (A) $\mathrm{mPEG}_{2000}-\mathrm{OH}$ and $\mathrm{mPEG}_{2000}-\mathrm{NH}_{2}$, (B) $\mathrm{HO}-\mathrm{PEG}_{2050}-\mathrm{OH}$ and $\mathrm{H}_{2} \mathrm{~N}-\mathrm{PEG}_{2050}-\mathrm{NH}_{2}$, and (C) $4 \mathrm{aPEG}_{2000}-\mathrm{OH}$ and $4 \mathrm{aPEG}_{2000}-\mathrm{NH}_{2}$.
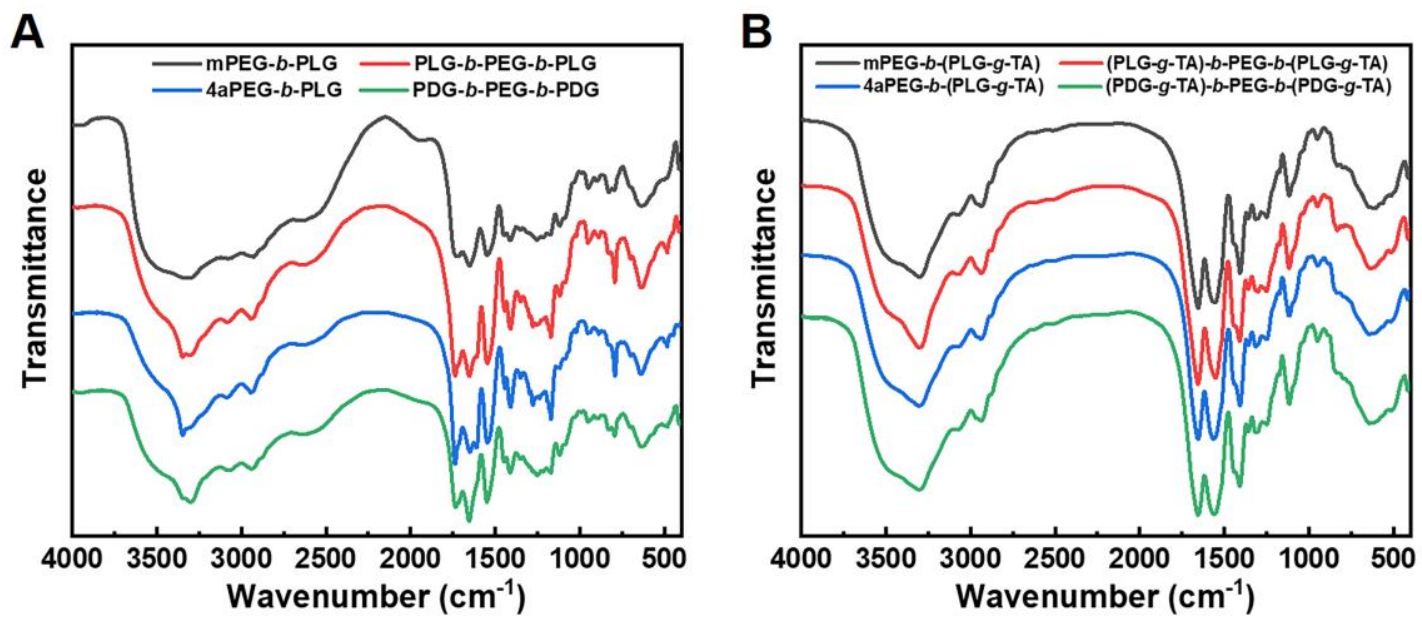

Figure S2. FT-IR spectra of (A) PEG-b-PGA precursor copolymers, and (B) PEG/(PGA-g-TA) copolymers. 


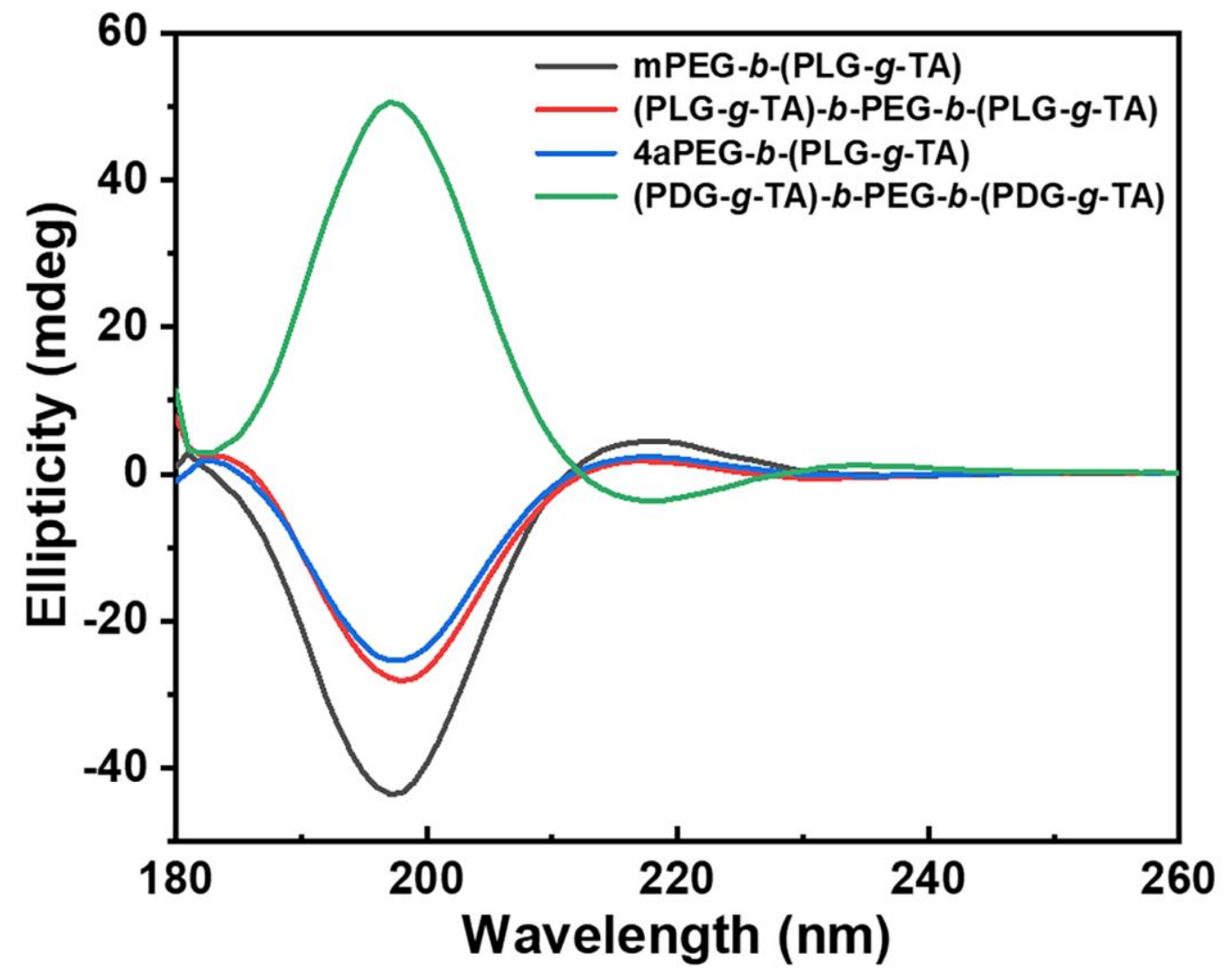

Figure S3. CD spectra of PEG/(PGA-g-TA) precursor copolymers at the concentration of 0.25 $\mathrm{mg} / \mathrm{mL}$ at $25^{\circ} \mathrm{C}$. 
Table S1. The G' at plateau of hydrogel samples of PEG/(PGA-g-TA) block copolymers with different concentrations of $\mathrm{H}_{2} \mathrm{O}_{2}$.

\begin{tabular}{ccccc}
\hline Concentrations of $\mathbf{H}_{\mathbf{2}} \mathbf{O}_{\mathbf{2}}(\mathbf{m M})$ & $\mathbf{0 . 9 1}$ & $\mathbf{1 . 8 1}$ & $\mathbf{2 . 7 3}$ & $\mathbf{3 . 8 3}$ \\
\hline mPEG- $b$-(PLG- $g$-TA) & $43 \mathrm{~Pa}$ & $3430 \mathrm{~Pa}$ & $8000 \mathrm{~Pa}$ & $9500 \mathrm{~Pa}$ \\
(PLG- $g$-TA)- $b$-PEG- $b$-(PLG- $g$-TA) & $5.3 \mathrm{~Pa}$ & $2470 \mathrm{~Pa}$ & $6700 \mathrm{~Pa}$ & $9840 \mathrm{~Pa}$ \\
4aPEG- $b$-(PLG- -TA) & $111 \mathrm{~Pa}$ & $3770 \mathrm{~Pa}$ & $8600 \mathrm{~Pa}$ & $11400 \mathrm{~Pa}$ \\
(PDG- $g$-TA)- $b$-PEG- $b$-(PDG- $g$-TA) & $221 \mathrm{~Pa}$ & $2580 \mathrm{~Pa}$ & $4100 \mathrm{~Pa}$ & $4430 \mathrm{~Pa}$ \\
\hline
\end{tabular}

The concentrations of copolymers, and HRP were $2 \%(\mathrm{w} / \mathrm{v})$, and $2 \mathrm{U} / \mathrm{mL}$, respectively. 


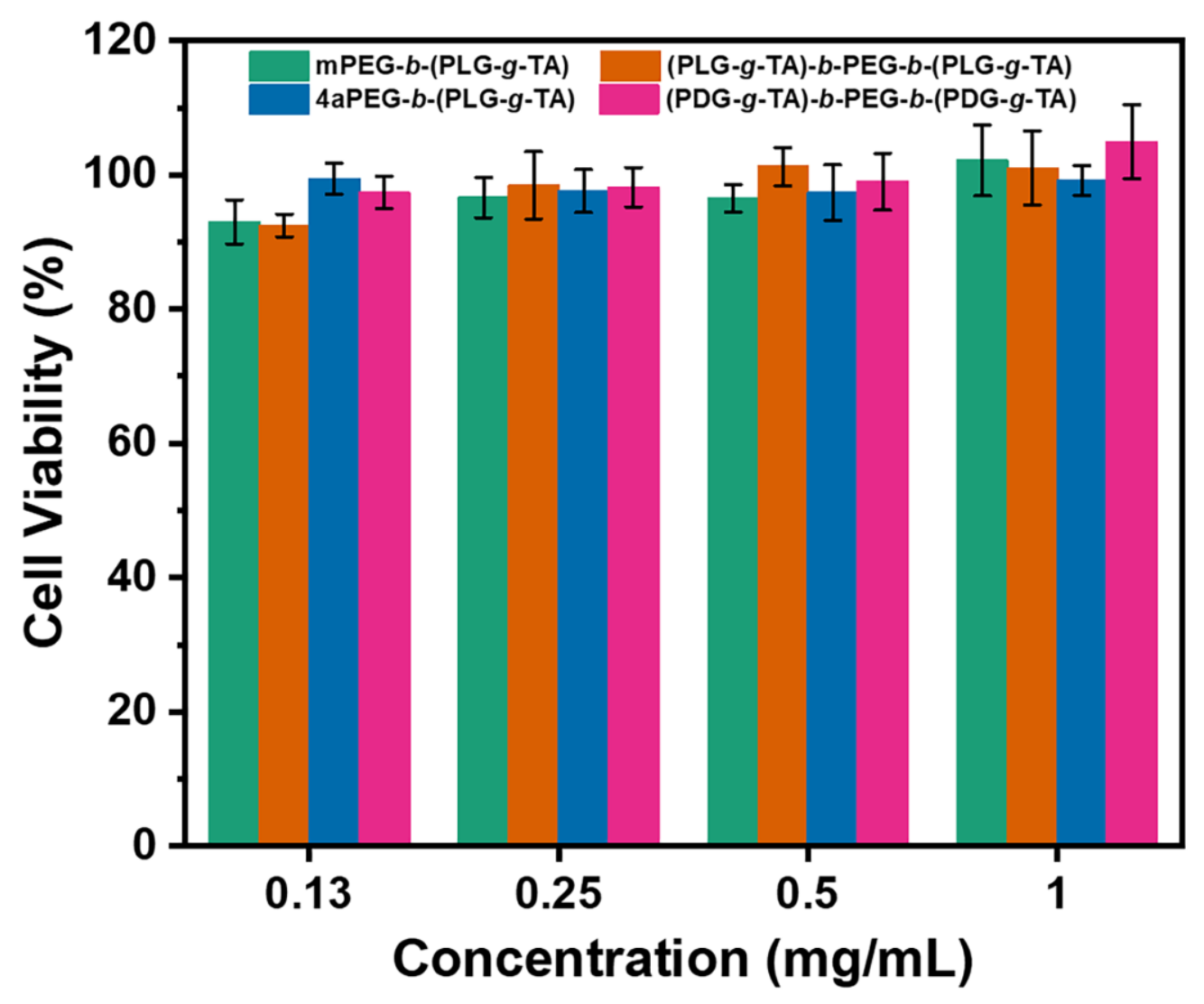

Figure S4. In vitro cytocompatibility L929 cells measured with CCK-8 after cultured with four types of PEG/(PGA-g-TA) precursor copolymers for $24 \mathrm{~h}(\mathrm{n}=4)$. 

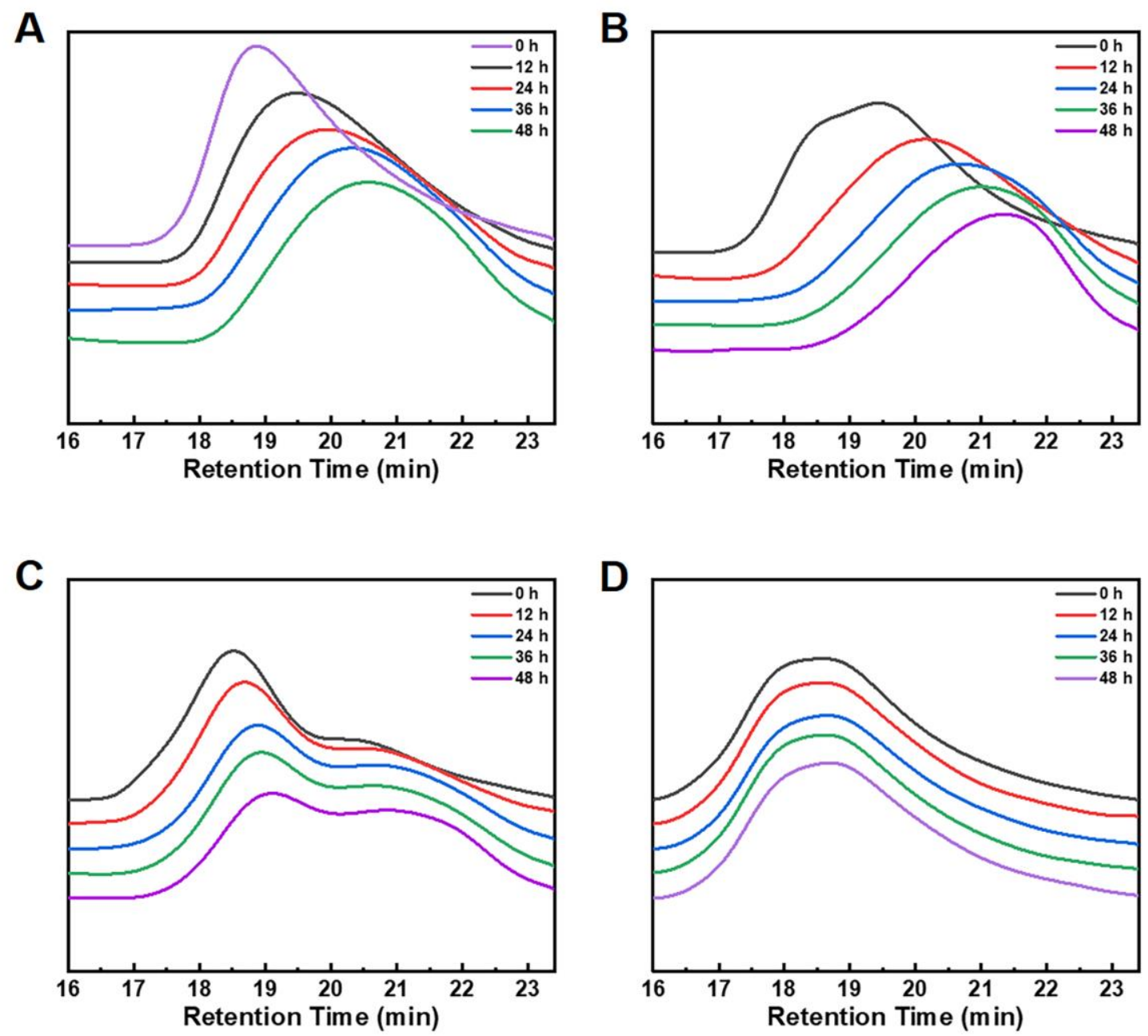

Figure S5. GPC traces of (A) mPEG- $b$-(PLG- $g$-TA), (B) (PLG- $g$-TA)- $b$-PEG- $b$-(PLG- $g$-TA), (C) 4aPEG- $b$-(PLG- $g$-TA), and (D) (PDG- $g$-TA)- $b$-PEG- $b$-(PDG- $g$-TA) after being incubated in PBS with elastase $(5 \mathrm{U} / \mathrm{mL})$ for different time intervals. 

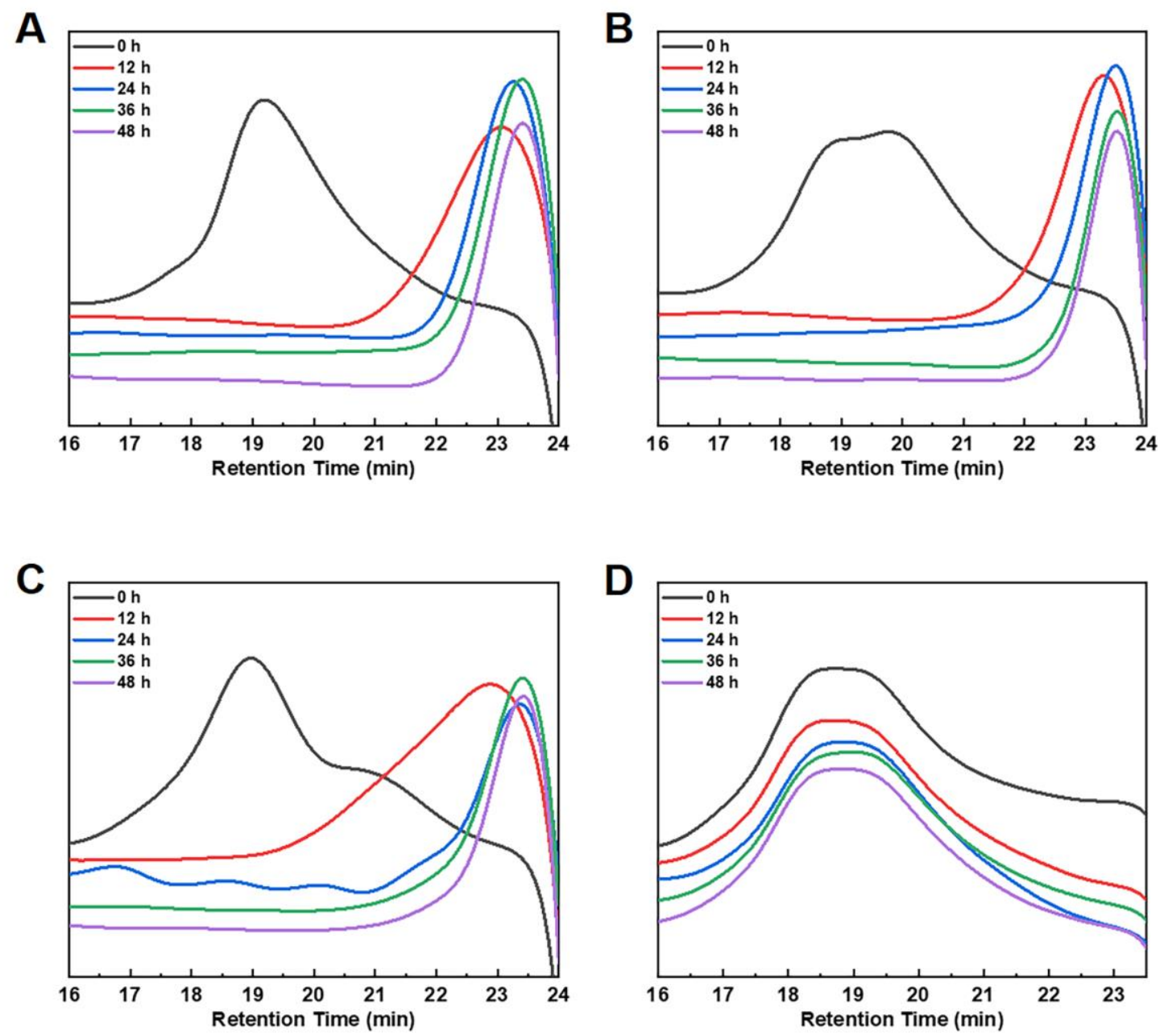

Figure S6. GPC traces of (A) mPEG- $b$-(PLG- $g$-TA), (B) (PLG- $g$-TA)- $b$-PEG- $b$-(PLG- $g$-TA), (C) 4aPEG- $b$-(PLG- $g$-TA), and (D) (PDG- $g$-TA)- $b$-PEG- $b$-(PDG- $g$-TA) after being incubated in PBS with proteinase $\mathrm{K}(5 \mathrm{U} / \mathrm{mL})$ for different time intervals. 


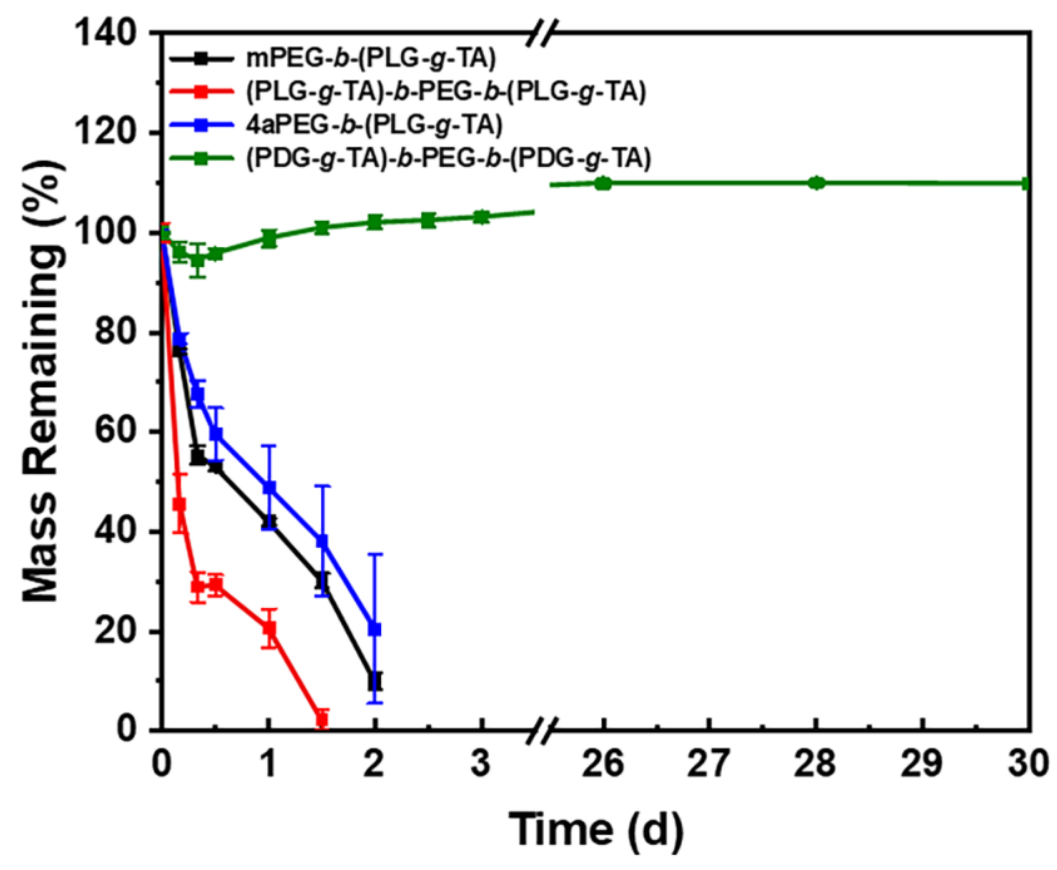

Figure S7. In vitro degradation profiles of hydrogel samples of four types of copolymers in PBS containing proteinase $\mathrm{K}(1 \mathrm{U} / \mathrm{mL})$. The concentrations of copolymers, $\mathrm{H}_{2} \mathrm{O}_{2}$, and HRP were $2 \%$ (w/v), $1.81 \mathrm{mM}$ and $2 \mathrm{U} / \mathrm{mL}$, respectively. 
A

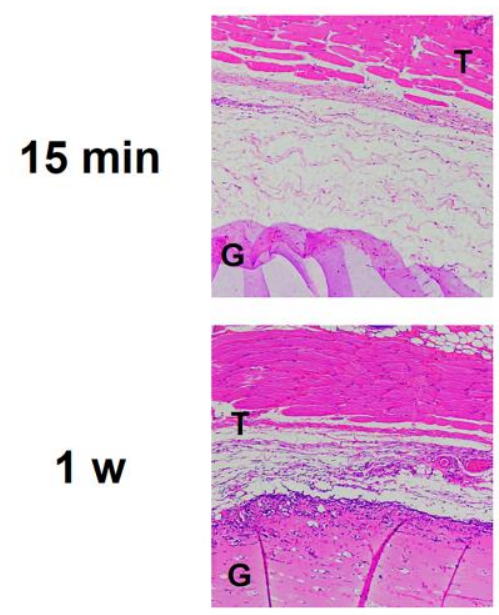

2 w

4 w
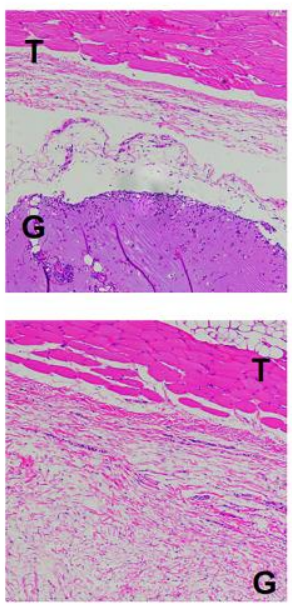

6 w
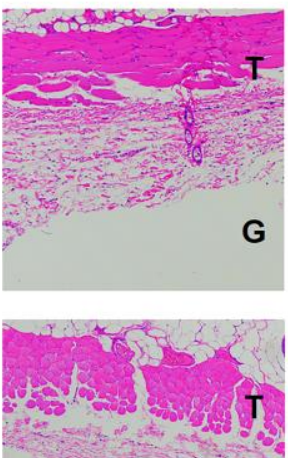

$8 w$

G

11 w
B
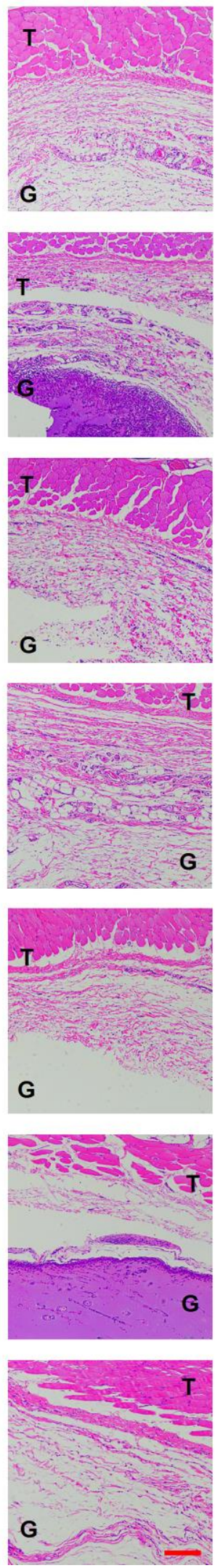

C
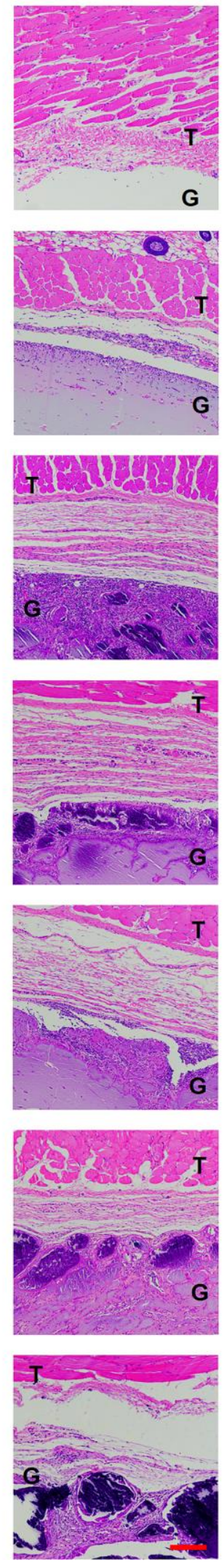

D
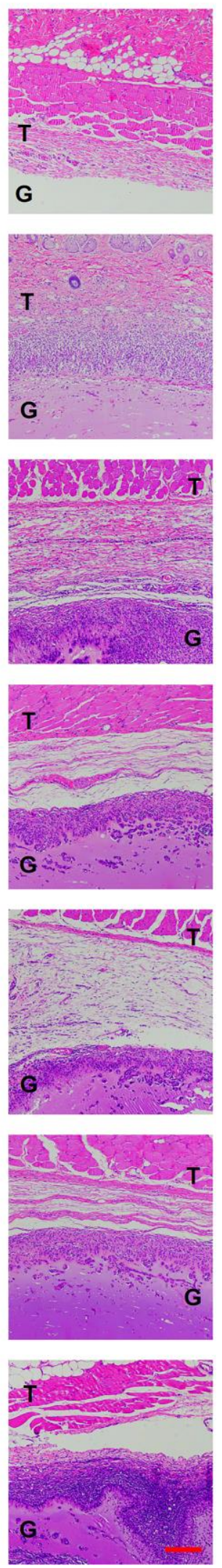
Figure S8. Images of H\&E-stained sections of tissue surrounding the injected (A) mPEG- $b$ (PLG- $g$-TA), (B) (PLG- $g$-TA)- $b$-PEG- $b$-(PLG- $g$-TA), (C) 4aPEG- $b$-(PLG- $g$-TA), and (D) (PDG- $g$-TA)- $b$-PEG- $b$-(PDG- $g$-TA) hydrogels at different time points. T: tissue, G: gel. Scale bars $=200 \mu \mathrm{m}$. 